\section{Aspectos psicossociais do trabalho e dor musculoesquelética em professores}

\author{
Psychosocial work-related factors and \\ musculoskeletal pain among schoolteachers
}

\author{
${ }^{1}$ Universidade Estadual do \\ Sudoeste da Bahia, Jequié, \\ Brasil. \\ 2 Departamento de Saúde, \\ Universidade Estadual de \\ Feira de Santana, Salvador, \\ Brasil. \\ 3 Faculdade de Medicina da \\ Bahia, Universidade Federal \\ da Bahia, Salvador, Brasil. \\ Correspondência \\ J. P. Cardoso \\ Universidade Estadual do \\ Sudoeste da Bahia. \\ Rua José Moreira Sobrinho $s / n$, \\ Jequié, BA 45206-190, Brasil. \\ jpcardoso@uesb.edu.br
}

\begin{abstract}
This study aimed to investigate the association between work-related psychosocial factors and musculoskeletal pain (MP) among primary schoolteachers in the public school system in Salvador, Bahia State, Brazil. A cross-sectional epidemiological study included all 4,496 teachers from the system. A self-applied mail questionnaire was used to collect information on complaints of musculoskeletal pain in the upper limbs, lower limbs, and back (dependent variables), demand-control model quadrants (independent variables), and covariables. Multivariate logistic regression showed that teachers in high-stress jobs presented the highest MP rates, and that those with low-stress jobs had the lowest rates in all three body segments. Teachers in active jobs presented higher MP rates than those in low-stress jobs, particularly in the upper limbs and back. Higher MP prevalence rates were associated with heavier psychological demand in all three body segments and with limited job control in the upper limbs only.
\end{abstract}

Musculoskeletal System; Pain; Faculty; Occupational Health
Jefferson Paixão Cardoso 1

Tânia Maria de Araújo 2

Fernando Martins Carvalho 3

Nelson Fernandes de Oliveira 2

Eduardo José Farias Borges dos Reis 3

\section{Introdução}

A relação entre os aspectos psicossociais do trabalho e a dor musculoesquelética tem sido estudada em diversas categorias profissionais 1,2,3, inclusive a docente 4,5 . A sobrecarga de trabalho, em conjunto com os aspectos psicossociais desfavoráveis do trabalho, podem acarretar desenvolvimento ou agravamento de quadros álgicos em segmentos corporais específicos como braços, pernas e coluna ${ }^{6}$. Estudos em diversos grupos ocupacionais encontraram associação entre os aspectos psicossociais e a dor musculoesquelética nos membros superiores 6,7,8,9, membros inferiores 8 e dorso 10,11.

As duas dimensões do Modelo DemandaControle 12 (demanda psicológica do trabalho e o controle sobre tarefas), assim como a combinação dessas dimensões (trabalho de alta exigência, trabalho ativo, trabalho passivo e trabalho de baixa exigência), demonstraram-se associadas à ocorrência de queixas de dor musculoesquelética em algumas categorias ocupacionais 8,11,13. No contexto brasileiro, estudos sobre os aspectos psicossociais do trabalho docente e dor musculoesquelética são escassos. Entretanto, a dor musculoesquelética é um problema relevante em professores, podendo comprometer a atividade laborativa, reduzir o desempenho ou levar ao afastamento das atividades do trabalho 14 .

Este estudo objetivou investigar a associação entre aspectos psicossociais do trabalho e a ocor- 
rência de dor musculoesquelética entre professores da rede municipal de ensino de Salvador, Bahia, Brasil.

\section{Metodologia}

Entre março e abril de 2006, a Secretaria Municipal de Educação e Cultura (SMEC) realizou um censo de professores da rede municipal de ensino infantil e fundamental da cidade de Salvador. Foram investigados 4.496 (95,7\%) professores, dentre os 4.697 elegíveis, de todos os tipos de vínculo de trabalho (permanente e temporário).

Um questionário padronizado foi respondido pelo professor, na escola em que trabalhava. $\mathrm{O}$ questionário incluiu questões sobre características sociodemográficas, características da atividade docente, características do ambiente de trabalho, dimensões psicossociais do trabalho e saúde do docente: queixas autorreferidas de nervosismo, sonolência, dor nos braços, dor nas pernas, dor nas costas, dor na garganta, fraqueza e falta de ar, entre outras.

O questionário foi entregue ao professor em envelope lacrado e não identificado durante o processo de recadastramento dos professores da rede municipal de ensino de Salvador. O envelope, além do questionário, continha uma carta do Secretário da Educação e Cultura, informando que a pesquisa era de caráter voluntário, com o pedido de que o professor não se identificasse. O projeto foi aprovado pelo Comitê de Ética em Pesquisa da Maternidade Climério de Oliveira da Universidade Federal da Bahia, parecer $n^{\circ}$. 83/2007.

O presente estudo analisa o vasto material produzido pelo estudo de corte transversal de caráter censitário realizado pela SMEC.

A variável independente foi constituída pelos aspectos psicossociais do trabalho, avaliados segundo o Modelo Demanda-Controle 15, considerando o controle do professor sobre o próprio trabalho e as demandas psicológicas envolvidas na atividade docente. Utilizou-se a versão do Job Content Questionnaire (JCQ) traduzida e validada 16 para grupos ocupacionais do Brasil.

A construção dos quadrantes propostos no modelo demanda-controle foi realizada com base nas respostas às questões dos blocos de controle sobre o trabalho e demanda psicológica do JCQ. Os itens de ambos os indicadores foram avaliados por escala que variou de 1 (discordo fortemente) a 4 (concordo fortemente). Procedeu-se ao cálculo de indicadores de "Controle" e "Demanda Psicológica", seguindo-se os procedimentos recomendados por Karasek 17 . Em seguida, as variáveis foram dicotomizadas em dois níveis: baixo e alto. O ponto de corte adotado foi a média de cada variável. Finalmente, foram construídas as categorias de análise do modelo, com base na combinação das variáveis controle e demanda, formando as quatro categorias de análise: alta exigência (baixo controle/ alta demanda), trabalho ativo (alto controle/alta demanda), trabalho passivo (baixo controle/baixa demanda) e baixa exigência (alto controle/ baixa demanda).

Os docentes expostos a uma alta demanda e baixo controle (trabalho de alta exigência) foram considerados como grupo de maior exposição. Aqueles expostos a baixa demanda psicológica e alto controle (baixa exigência) foram classificados como grupo não exposto e utilizado como grupo de referência nas análises.

A variável dependente foi a dor musculoesquelética autorreferida, analisada quanto à frequência e topografia (dor nos braços: membros superiores; dor nas pernas: membros inferiores; e dor nas costas: dorso). Considerou-se como "caso" o professor que referiu dor musculoesquelética "frequente" ou "muito frequente", numa escala que ainda incluía as opções "nunca", "raramente" e "pouco frequente".

Para avaliação de possível efeito sobre a associação principal investigada foram analisadas covariáveis: sociodemográficas (sexo, idade, nível de escolaridade, situação conjugal, número de filhos); relativas ao trabalho docente (tempo de trabalho na profissão, tempo de trabalho na escola, turnos de trabalho na escola, nível das turmas a que ensinava, número de turmas e de alunos, carga horária semanal, trabalho em outra escola dentro e fora da rede municipal, outra atividade remunerada e esforço físico excessivo).

A medida de associação utilizada foi a razão de prevalências (RP) e seus respectivos intervalos de 95\% de confiança (IC95\%).

Foram utilizadas técnicas de análise de regressão logística 18 . Foi desenvolvido um modelo para cada segmento corporal (membros inferiores, membros superiores e dorso), ajustado segundo covariáveis relevantes. Inicialmente, foi realizada pré-seleção das variáveis para inclusão no modelo de regressão logística, adotando-se um valor de $\mathrm{p} \leq 0,25$. A modificação de efeito foi analisada quanto à significância estatística dos termos de interação pelo teste de razão de verossimilhança, no nível de $20 \%$. Assim, procedeu-se à análise simultânea de todas as variáveis candidatas à modificação de efeito e quando o valor do teste era abaixo do valor crítico, avaliou-se o efeito de cada variável separadamente.

Para análise das variáveis potencialmente confundidoras, foi verificada a magnitude da variação dos coeficientes estimados da variável 
de exposição principal (modelo demanda-controle). Para isso, o modelo com todas as variáveis preditoras foi comparado ao modelo com a retirada de uma preditora. A variável foi considera confundidora quando apresentou variação superior a $10 \%$ nas categorias de alta exigência e trabalho ativo. Verificada a presença de confundimento, o modelo final foi ajustado pelas variáveis confundidoras.

Considerando que a prevalência de dor musculoesquelética foi elevada na população de professores, foram estimadas as RP e calculados os IC95\%, utilizando-se o método Delta 19.

Para avaliação da bondade do ajuste do modelo foi utilizado o teste de Le Cessie-van Houwelingen-Copas-Hosmer ${ }^{20}$. A área sobre a curva ROC (Receiver Operating Characteristic) foi utilizada para discriminação do modelo. A análise da influência dos padrões de covariáveis foi realizada comparando-se a estimativa dos parâmetros obtidos pela eliminação dos padrões de covari- áveis com a estimativa dos parâmetros obtidos mantendo-se os padrões de covariáveis.

Os dados foram analisados com o auxílio do programa estatístico SPSS, versão 9.0 (SPSS Inc., Chicago, Estados Unidos), e R, versão 2.10.0 (The R Foundation for Statistical Computing, Viena, Áustria; http://www.r-project.org).

\section{Resultados}

Dos 4.496 professores investigados, 1.299 foram excluídos da análise porque não responderam a alguma das questões das escalas de controle e demanda do JCQ.

As distribuições de diversas variáveis sociodemográficas (Tabela 1) e relativas ao trabalho docente (Tabela 2) dos 3.197 professores com informações disponíveis sobre as escalas do JCQ foram semelhantes às dos 1.299 professores para os quais estas informações não estavam

Tabela 1

Variáveis sociodemográficas segundo disponibilidade de informações sobre o Modelo Demanda-Controle em professores de Salvador, Bahia, Brasil, 2006.

\begin{tabular}{|c|c|c|c|c|c|}
\hline \multirow[t]{2}{*}{ Variável } & \multicolumn{2}{|c|}{ Informações disponíveis } & \multicolumn{2}{|c|}{ Informações não disponíveis } & \multirow[t]{2}{*}{ Valor de $p$} \\
\hline & $\mathrm{n}$ & $\%$ & n & $\%$ & \\
\hline Sexo & & & & & $<0,050$ \\
\hline Feminino & 2.833 & 91,4 & 1.161 & 93,4 & \\
\hline Masculino & 266 & 8,6 & 82 & 6,6 & \\
\hline Faixa etária (anos) & & & & & $<0,001$ \\
\hline Até 29 & 469 & 15,2 & 137 & 11,3 & \\
\hline $30-39$ & 1.226 & 39,7 & 408 & 33,7 & \\
\hline$\geq 40$ & 1.395 & 45,1 & 667 & 55,0 & \\
\hline Escolaridade & & & & & $<0,001$ \\
\hline Médio & 821 & 26,1 & 449 & 35,7 & \\
\hline Superior & 2.319 & 73,9 & 809 & 64,3 & \\
\hline Situação conjugal & & & & & 0,108 \\
\hline Solteiro & 1.232 & 39,7 & 445 & 36,4 & \\
\hline Casado & 1.454 & 46,8 & 594 & 48,6 & \\
\hline Viúvo/Separado/Divorciado & 418 & 13,5 & 183 & 15,0 & \\
\hline Tem filhos & & & & & $<0,010$ \\
\hline Sim & 1.982 & 62,5 & 850 & 66,8 & \\
\hline Não & 1.189 & 37,5 & 423 & 33,2 & \\
\hline Número de filhos & & & & & $<0,010$ \\
\hline 1 & 823 & 41,8 & 311 & 37,0 & \\
\hline 2 & 736 & 37,3 & 310 & 36,9 & \\
\hline$\geq 3$ & 411 & 20,9 & 219 & 26,1 & \\
\hline Tempo de trabalho como professor (anos) & & & & & $<0,001$ \\
\hline$\leq 14$ & 1.610 & 54,7 & 522 & 45,5 & \\
\hline$>14$ & 1.333 & 45,3 & 625 & 54,5 & \\
\hline
\end{tabular}


Variáveis relativas ao trabalho docente segundo disponibilidade de informações sobre o Modelo Demanda-Controle em professores de Salvador, Bahia, Brasil, 2006

\begin{tabular}{|c|c|c|c|c|c|}
\hline \multirow[t]{2}{*}{ Variável } & \multicolumn{2}{|c|}{ Informações disponíveis } & \multicolumn{2}{|c|}{ Informações não disponíveis } & \multirow[t]{2}{*}{ Valor de $\mathrm{p}$} \\
\hline & $\mathbf{n}$ & $\%$ & $\mathbf{n}$ & $\%$ & \\
\hline Tempo de trabalho na escola (anos) & & & & & 0,010 \\
\hline$\leq 5$ & 1.611 & 60,7 & 590 & 55,6 & \\
\hline$>5$ & 1.047 & 39,4 & 471 & 44,4 & \\
\hline Turnos de trabalho na escola & & & & & 0,453 \\
\hline 1 & 1.442 & 46,1 & 593 & 47,4 & \\
\hline$\geq 2$ & 1.685 & 53,9 & 659 & 52,6 & \\
\hline Nível das turmas que ensina & & & & & 0,833 \\
\hline 1 & 2.569 & 93,7 & 1.008 & 93,5 & \\
\hline$\geq 2$ & 173 & 6,3 & 70 & 6,5 & \\
\hline Número de turmas que ensina & & & & & 0,895 \\
\hline 1 & 1.221 & 45,5 & 473 & 44,9 & \\
\hline 2 & 1.065 & 39,7 & 418 & 39,7 & \\
\hline$\geq 3$ & 400 & 14,8 & 163 & 15,5 & \\
\hline Número de alunos por turma & & & & & 0,001 \\
\hline$\leq 30$ & 1.548 & 54,3 & 516 & 47,3 & \\
\hline$>30$ & 1.302 & 45,7 & 575 & 52,7 & \\
\hline Carga horária semanal (horas) & & & & & 0,447 \\
\hline 20 & 1.330 & 45,5 & 532 & 46,9 & \\
\hline 40 & 1.590 & 54,5 & 603 & 53,1 & \\
\hline $\begin{array}{l}\text { Trabalho em outra escola da rede } \\
\text { municipal }\end{array}$ & & & & & 0,348 \\
\hline $\operatorname{Sim}$ & 449 & 14,5 & 165 & 13,4 & \\
\hline Não & 2.646 & 85,5 & 1.066 & 86,6 & \\
\hline $\begin{array}{l}\text { Trabalho em outra escola fora da rede } \\
\text { municipal }\end{array}$ & & & & & 0,423 \\
\hline $\operatorname{Sim}$ & 924 & 31,6 & 374 & 32,9 & \\
\hline Não & 2.001 & 68,4 & 763 & 67,1 & \\
\hline Outra atividade remunerada & & & & & 0,050 \\
\hline Sim & 314 & 11,2 & 124 & 11,4 & \\
\hline Não & 2.492 & 88,8 & 968 & 88,6 & \\
\hline Muito esforço físico & & & & & 0,050 \\
\hline Não & 1.746 & 54,9 & 646 & 58,9 & \\
\hline Sim & 1.433 & 45,1 & 451 & 41,1 & \\
\hline
\end{tabular}

disponíveis. Apenas a variável "situação conjugal” não apresentou diferenças estatisticamente significativas entre respondentes e não respondentes do JCQ. Contudo as variáveis ocupacionais "tempo de trabalho na escola", "número de alunos por turma”, "outra atividade remunerada" e "muito esforço físico" configuraram diferenças estatísticas.

As prevalências de dor musculoesquelética (DME) foram $41,1 \%$ para membros inferiores, $41,1 \%$ no dorso e $23,7 \%$ para os membros superiores.
As Tabelas 3, 4 e 5 apresentaram a associação bruta e ajustada entre DME e aspectos psicossociais do trabalho (Demanda, Controle e quadrantes do Modelo D-C), nos três segmentos corporais estudados (membros inferiores, membros superiores e dorso). A análise de modificação de efeito não identificou interação entre as variáveis analisadas. De uma forma geral, os ajustes pelas respectivas variáveis confundidoras não alteraram substancialmente os valores das RPs brutas. A maior diferença obtida para a RP após ajuste foi inferior a $8 \%$ do valor da RP bruta. 
Prevalências (\%), razões de prevalências (RP) * e seus respectivos intervalos de 95\% de confiança (IC95\%) de dor musculoesquelética em membros inferiores ** segundo grupos do Modelo Demanda-Controle em professores de Salvador, Bahia, Brasil, 2006.

\begin{tabular}{|c|c|c|c|}
\hline \multirow[t]{2}{*}{ Variáveis } & \multicolumn{3}{|c|}{ Dor em membros inferiores $(n=3.110)$} \\
\hline & $\%$ & $\mathrm{RP}_{\text {bruta }}(\mathrm{IC} 95 \%)$ & $\mathrm{RP}_{\text {ajustada }}(\mathrm{IC} 95 \%)$ \\
\hline \multicolumn{4}{|c|}{ Modelo Demanda-Controle } \\
\hline Baixa exigência & 38,1 & 1,00 & 1,00 \\
\hline Trabalho passivo & 36,9 & $0,97(0,85-1,09)$ & $0,96(0,85-1,08)$ \\
\hline Trabalho ativo & 44,5 & 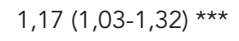 & $1,13(0,99-1,29)$ \\
\hline Alta exigência & 46,1 & $1,21(1,08-1,35) \#$ & $1,14(1,04-1,29) * \star \star$ \\
\hline \multicolumn{4}{|l|}{ Sexo } \\
\hline Masculino & 22,0 & 1,00 & 1,00 \\
\hline Feminino & 42,6 & $1,98(1,57-2,38) \# \#$ & $2,04(1,60-2,59) \# \#$ \\
\hline \multicolumn{4}{|l|}{ Faixa etária (anos) } \\
\hline$\leq 39$ & 38,6 & 1,00 & 1,00 \\
\hline$>39$ & 43,7 & $1,13(1,05-1,22) \#$ & $1,20(1,10-1,31) \#$ \\
\hline \multicolumn{4}{|c|}{ Esforço físico excessivo } \\
\hline Sim & 34,7 & 1,00 & 1,00 \\
\hline Não & 49,3 & $1,42(1,32-1,52) \#$ & $1,39(1,27-1,52) \#$ \\
\hline 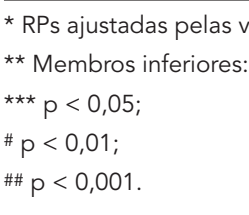 & $\begin{array}{l}\text { nos re } \\
\text { esforç }\end{array}$ & $\begin{array}{l}\text { delos finais de regressã } \\
\text { vo; }\end{array}$ & tica múltipla; \\
\hline
\end{tabular}

Os resultados evidenciaram forte associação estatística entre demanda psicológica do trabalho e DME nos três segmentos estudados. A prevalência de DME nos membros superiores foi 1,36 vez maior em professores com alta demanda, após ajustes (Tabela 4).

O controle sobre o trabalho estava associado com a dor musculoesquelética apenas nos membros superiores (RP = 1,18; IC95\%: 1,03-1,35).

A prevalência de DME foi mais elevada nos professores do grupo com trabalho de alta exigência que nos professores dos demais quadrantes, nos três segmentos corporais estudados, mesmo após os ajustes por potenciais confundidores. A prevalência de DME em membros superiores (Tabela 4) foi 1,56 vez maior nos professores em alta exigência $(29,8 \%)$ do que entre aqueles com baixa exigência (19,1\%).

A prevalência de DME em professores em trabalho ativo estava mais elevada do que a de professores em trabalhos de baixa exigência, especialmente nos segmentos membros superiores $(\mathrm{RP}=1,37 ; 1,1-1,71)$ e dorso $(\mathrm{RP}=1,20$; 1,05-1,34).

A análise do ajustamento dos modelos de regressão logística, feita pelo teste de Le Cessie-van
Houwelingen-Copas-Hosmer, indicou que os modelos obtidos ajustaram-se satisfatoriamente aos dados. O valor da área sob a curva ROC para cada modelo estudado demonstrou razoável discriminação entre os docentes com e sem a dor musculoesquelética.

No diagnóstico dos modelos de regressão logística, a exclusão dos valores dos padrões das covariáveis mais influentes, superiores ao valor crítico 4 dos diagnósticos da redução de $\chi^{2}$ de Pearson $\left(\Delta \chi^{2}\right)$, não provocou alterações significantes nos coeficientes das variáveis dos modelos analisados.

\section{Discussão}

Inicialmente, é relevante considerar algumas possíveis limitações deste estudo de corte transversal que proporciona apenas uma visão instantânea da exposição e do efeito estudado. Portanto, não foi possível avaliar temporalmente a relação entre causa (aspectos psicossociais do trabalho) e efeito (dor musculoesquelética).

Não foi possível avaliar os professores afastados, os que abandonaram a profissão ou fale- 
Prevalências (\%), razões de prevalências (RP) * e seus respectivos intervalos de 95\% de confiança (IC95\%) de dor musculoesquelética em membros superiores ** segundo grupos do Modelo Demanda-Controle em professores de Salvador, Bahia, Brasil, 2006.

\begin{tabular}{|c|c|c|c|}
\hline \multirow[t]{2}{*}{ Variáveis } & \multicolumn{3}{|c|}{ Dor em membros superiores $(n=3.071)$} \\
\hline & $\%$ & $\mathrm{RP}_{\text {bruta }}(\mathrm{IC} 95 \%)$ & $\mathrm{RP}_{\text {ajustada }}(\mathrm{IC} 95 \%)$ \\
\hline \multicolumn{4}{|c|}{ Modelo Demanda-Controle } \\
\hline Baixa exigência & 19,1 & 1,00 & 1,00 \\
\hline Trabalho passivo & 21,7 & $1,14(0,94-1,37)$ & $1,09(0,89-1,35)$ \\
\hline Trabalho ativo & 26,1 & 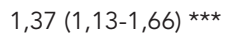 & $1,37(1,11-1,71) * * *$ \\
\hline Alta exigência & 29,8 & $1,56(1,31-1,87) \#$ & $1,58(1,30-1,93) \#$ \\
\hline \multicolumn{4}{|l|}{ Sexo } \\
\hline Masculino & 15,4 & 1,00 & 1,00 \\
\hline Feminino & 24,6 & $1,59(1,22-2,07) \#$ & $1,67(1,21-2,30) \#$ \\
\hline \multicolumn{4}{|l|}{ Faixa etária (anos) } \\
\hline$\leq 39$ & 20,7 & 1,00 & 1,00 \\
\hline$>39$ & 27,7 & $1,33(1,19-1,49)$ \# & $1,45(1,26-1,67) \#$ \\
\hline \multicolumn{4}{|c|}{ Esforço físico excessivo } \\
\hline $\operatorname{Sim}$ & 20,1 & 1,00 & 1,00 \\
\hline Não & 28,6 & $1,42(1,27-1,59) \#$ & $1,28(1,11-1,48) \#$ \\
\hline \multicolumn{4}{|c|}{ Número de alunos por turma } \\
\hline$\leq 30$ & 22,3 & 1,00 & 1,00 \\
\hline$>30$ & 25,6 & $1,14(1,02-1,28) \# \#$ & $1,14(1,00-1,32)$ \\
\hline
\end{tabular}

* RPs ajustadas pelas variáveis que permaneceram nos respectivos modelos finais de regressão logística múltipla;

** Membros superiores: ajustado por sexo, idade, número de alunos e esforço físico excessivo;

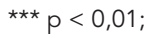

$\# p<0,0001$;

$\#$ \# $<<0,05$.

cidos. Ao considerar apenas docentes no exercício atual da profissão, os dados poderiam subestimar a prevalência de dor musculoesquelética, por conta do Efeito do Trabalhador Sadio.

Outra limitação refere-se à localização e à frequência de dor musculoesquelética autorreferida. A localização do evento englobou segmentos corporais amplos, não diferenciando subconjuntos que poderiam elucidar, com maior precisão, o verdadeiro sítio de ocorrência da dor. Contudo, registram-se na literatura estudos que utilizaram instrumentos de coleta autorreferidos para investigar dor musculoesquelética $6,7,21$, reforçando a pertinência e utilidade de tal procedimento.

Não foi investigada a duração da ocorrência de DME em períodos anteriores à coleta de dados. Mas, no caso específico das exigências no trabalho, é pouco provável que tenha ocorrido causalidade reversa (deslocamento de professores com dor musculoesquelética para o trabalho de alta exigência).

Tanto as variáveis do modelo demanda-controle como as ocorrências de dor musculoesque- lética estiveram sujeitas aos efeitos da sazonalidade do ano letivo. O maior acúmulo de tarefas docentes acontece no final do ano letivo, o que poderia subestimar ou superestimar o efeito pesquisado, a depender do período da coleta de dados 22. Assim, os professores estudados nessa pesquisa estariam enfrentando uma menor sobrecarga de atividades, pois a coleta de dados foi realizada no meio do primeiro semestre letivo (março/abril).

A proporção de professores que responderam às escalas de demanda e controle do JCQ foi razoável: $71 \%$. A comparação das proporções dos indivíduos incluídos e excluídos na análise do Modelo Demanda-Controle indicou semelhanças. Assim, a exclusão dos registros por respostas incompletas, muito provavelmente, não enviesou os resultados.

Apesar de suas possíveis limitações descritas aqui, o estudo avaliou um contingente expressivo de professores, possibilitando investigar condições do trabalho docente e efeitos adversos à saúde. 
Prevalências (\%), razões de prevalências (RP) * e seus respectivos intervalos de 95\% de confiança (IC95\%) de dor musculoesquelética no dorso ** segundo grupos do Modelo Demanda-Controle em professores de Salvador, Bahia, Brasil, 2006.

\begin{tabular}{|c|c|c|c|}
\hline \multirow[t]{2}{*}{ Variáveis } & \multicolumn{3}{|c|}{ Dor no dorso $(n=3.109)$} \\
\hline & $\%$ & $\mathrm{RP}_{\text {bruta }}(\mathrm{IC} 95 \%)$ & $\mathrm{RP}_{\text {ajustada }}(\mathrm{IC95 \% )}$ \\
\hline \multicolumn{4}{|c|}{ Modelo Demanda-Controle } \\
\hline Baixa exigência & 37,7 & 1,00 & 1,00 \\
\hline Trabalho passivo & 37,5 & $0,99(0,88-1,12)$ & $0,97(0,85-1,01)$ \\
\hline Trabalho ativo & 45,3 & 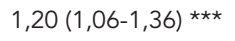 & $1,20(1,05-1,36)$ *** \\
\hline Alta exigência & 45,8 & $1,22(1,08-1,36) * \star \star$ & $1,19(1,05-1,34) * \star \star$ \\
\hline \multicolumn{4}{|l|}{ Sexo } \\
\hline Masculino & 26,6 & 1,00 & 1,00 \\
\hline Feminino & 42,1 & $1,58(1,31-1,90) \#$ & $1,75(1,40-2,18) \#$ \\
\hline \multicolumn{4}{|l|}{ Faixa etária (anos) } \\
\hline$\leq 39$ & 37,7 & 1,00 & 1,00 \\
\hline$>39$ & 44,6 & $1,18(1,10-1,27) \#$ & $1,22(1,12-1,33) \#$ \\
\hline \multicolumn{4}{|c|}{ Esforço físico excessivo } \\
\hline Sim & 36,4 & 1,00 & 1,00 \\
\hline Não & 47,0 & $1,29(1,20-1,38) \#$ & $1,25(1,14-1,37) \#$ \\
\hline
\end{tabular}

* RPs ajustadas pelas variáveis que permaneceram nos respectivos modelos finais de regressão logística múltipla;

** Dorso: ajustado por sexo, idade e esforço físico excessivo;

$\star \star \star * ~ p<0,01 ;$

$\# p<0,0001$.

As prevalências de DME nos membros superiores e dorso entre professores da rede municipal de ensino de Salvador foram elevadas: ambas $41,1 \%$, apontando que quase metade dos docentes apresentou dor nos segmentos corporais citados. Outros estudos que utilizaram instrumentos autorreferidos de condições de saúde e trabalho em professores encontraram prevalências semelhantes para DME 23,24,25. Num estudo com professores das escolas da rede particular de ensino de Salvador, as queixas de dor mais referidas foram dor nos membros inferiores $(47,1 \%)$ e costas (45\%) 23. A prevalência de dor em membros superiores referida por professores da rede particular de ensino de Vitória da Conquista foi de $52,1 \% 24$ e a prevalência de dor nas costas foi de $38,8 \%$, em professores universitários 25 .

Em outras categorias profissionais, consideradas potencialmente estressantes ou com altos níveis de estresse, como enfermeiras 2 , técnicos de radiologia 26 e médicos 27 , prevaleceram as DME na região lombar. Em membros superiores, costumam ser observadas elevadas prevalências de DME em trabalhadores do setor administrativo 6 e da indústria 28 .

Os aspectos psicossociais do trabalho associaram-se de maneira forte e positiva com DME. Professores expostos à alta exigência no trabalho e a trabalho ativo apresentaram maiores prevalências de DME nos três segmentos estudados, comparados aos professores em baixa exigência. Os resultados do presente estudo fortalecem o pressuposto do modelo de Karasek 12 que estabelece que o trabalho sob condições de baixo controle e alta demanda (alta exigência) é prejudicial à saúde. Assim, a associação positiva entre o trabalho de alta exigência e DME nos membros inferiores, membros superiores e dorso corrobora os achados de outros estudos que investigaram os aspectos psicossociais do trabalho (utilizando o modelo demanda-controle) e DME 3,7,8,9,21,29,30. Alguns estudos também encontraram associação entre trabalho ativo e DME 9,21.

O papel do esforço físico nas investigações sobre DME tem sido destacado por muitos autores $8,11,13$. Bongers et al. 31 discutem que as demandas físicas estariam numa rede intermediária e, ao mesmo tempo, confundindo a associação entre os aspectos psicossociais e as DME. Neste estudo não foi possível caracterizar o efeito de demandas físicas mais específicas da atividade docente, com a investigação de situações como permanência na posição em pé ou sentada, escrever no quadro, posicionamento inadequado do corpo, deslocamento segurando peso excessivo, entre outras situações que influenciariam a ocorrência 
de DME. Avaliou-se apenas um indicador global de carga física: o esforço físico. Cabe registrar que embora a variável esforço físico tenha permanecido nos modelos de análise selecionados para DME nas três regiões do corpo analisadas, mostrando ser um fator de risco independente para DME, esta não alterou a medida de associação entre os aspectos psicossociais do trabalho e DME mesmo após ajuste.

Os estudos que investigaram a influência dos fatores físicos e psicossociais na ocorrência das DME têm destacado o efeito potencializador promovido pela combinação desses dois fatores $6,30,31$. Investigações mais apuradas sobre o efeito da demanda, do controle e das cargas de trabalho (fatores físicos) na ocorrência de DME na população de professores estudada possibilitaria elucidar melhor a inter-relação entre diferentes características do trabalho e seus efeitos sobre a saúde, especialmente no que se refere à dor musculoesquelética.

Este estudo investigou uma população relativamente jovem, predominantemente feminina, com sobrecarga no trabalho, carga horária excessiva, trabalhando em mais de um turno e em trabalho de alta exigência. Os professores apresentaram prevalência elevada de DME nos membros superiores e nas costas. A situação de alta exigência e trabalho ativo destacaram-se como importantes preditores para ocorrência de DME nos segmentos analisados. Situações de trabalho desfavoráveis e a elevada prevalência da doença musculoesquelética poderão repercutir negativamente na qualidade de vida dos professores e na qualidade do ensino. Assim, torna-se necessária a busca de maior conhecimento, visando a adoção de políticas e ações para redução, prevenção e monitoramento desses acometimentos.

\section{Resumo}

Investigou-se a associação entre aspectos psicossociais do trabalho e queixas de dor musculoesquelética (DME) em professores. Um estudo censitário de corte transversal investigou 4.496 professores da rede municipal de ensino infantil e fundamental de Salvador, Bahia, Brasil. Informações sobre queixas de dor musculoesquelética em membros superiores, membros inferiores e dorso (variáveis dependentes), categorias do Modelo Demanda-Controle (variáveis independentes) e covariáveis foram coletadas num questionário padronizado autoaplicável. Análises de regressão logística multivariada revelaram que professores com trabalho de alta exigência apresentaram prevalência de DME mais elevada e professores em baixa exigência, prevalência mais baixa, em cada um dos três segmentos corporais estudados. Professores em trabalho ativo apresentaram prevalência de DME mais elevada do que professores em trabalho de baixa exigência, em membros superiores e dorso. Maiores taxas de prevalência de DME associaram-se à maior demanda psicológica nos três segmentos corporais e ao baixo controle sobre o trabalho em membros superiores, apenas.

Sistema Musculosquelético; Dor; Docentes; Saúde do Trabalhador

\section{Colaboradores}

T. M. Araújo, E. J. F. B. Reis e F. M. Carvalho participaram da concepção e da coordenação geral da pesquisa, do trabalho de campo, análise dos dados e redação do manuscrito. J. P. Cardoso trabalhou na revisão bibliográfica, supervisão da digitação, no processamento e análise dos dados e na redação do manuscrito. N. F. Oliveira trabalhou na análise de dados e na redação do manuscrito.

\section{Conflito de interesses}

T. M. Araújo e E. J. F. B. Reis são assessores do Sindicato dos Professores do Estado da Bahia (SINPRO-BA) que atende a docentes da rede particular de ensino. 


\section{Referências}

1. Menzel NN. Psychosocial factors in musculoskeletal disorders. Crit Care Nurs Clin N Am 2007; 19:445-53.

2. Smith DR, Wei N, Zhao L, Wang R-S. Musculoskeletal complaints and psychosocial risk factors among Chinese hospital nurses. Occup Med 2004; 54:579-82.

3. Wadman C, Kjellberg A. The role of the affective stress response as a mediator for the effect of psychosocial risk factors on musculoskeletal complaints - Part 2: hospital workers. Int J Ind Ergon 2007; 37:395-403.

4. Araújo TM, Carvalho FM. Condições de trabalho docente e saúde na Bahia: estudos epidemiológicos. Educação \& Sociedade 2009; 30:427-49.

5. Cardoso JP, Ribeiro QBR, Araújo TM, Carvalho FM, Reis EJFB. Prevalência de dor musculoesquelética em professores. Rev Bras Epidemiol 2009; 12: 604-14.

6. Devereux JJ, Vlachonikolis IG, Buckle PW. Epidemiological study to investigate potential interaction between physical and psychosocial factors at work that may increase the risk of symptoms of musculoskeletal disorder of the neck and upper limb. Occup Environ Med 2002; 59:269-77.

7. Leroux I, Brisson C, Montreuil S. Job strain and neck-shoulder symptoms: a prevalence study of women and men white-collar workers. Occup Med 2006; 56:102-9.

8. Leroux I, Dionne CE, Bourbonnais R, Brisson C. Prevalence of musculoskeletal pain and associated factors in the Quebec working population. Int Arch Occup Environ Health 2005; 8:379-86.

9. van den Heuvel SG, van der Beek AJ, Blatter BM, Hoogendoorn WE, Bongers PM. Psychosocial work characteristics in relation to neck and upper limb symptoms. Pain 2005; 114:47-53.

10. Andersen JH, Haahr JP, Frost P. Risk factors for more severe regional musculoskeletal symptoms: a two-year prospective study of a general working population. Arthritis Rheum 2007; 56:1355-64.

11. Ghaffari M, Alipour A, Farshad AA, Jensen I, Josephson M, Vingard E. Effect of psychosocial factors on low back pain in industrial workers. Occup Med 2008; 58:341-7.

12. Karasek RA. Job demand, job decision latitude, and mental strain: implications for job redesign. Administrative Science Quarterly 1979; 24:285-308.

13. Lee H, Wilbur J, Kim MJ, Miller AM. Psychosocial risk factors for work-related musculoskeletal disorders of the lower-back among long-haul international female flight attendants. J Adv Nurs 2008; 61:492-502.

14. Gasparini SM, Barreto SM, Assunção AA. O professor, as condições de trabalho e os efeitos sobre sua saúde. Educação e Pesquisa 2005; 31:189-99.

15. Araújo TM, Graça CC, Araújo E. Estresse ocupacional e saúde: contribuições do modelo demandacontrole. Ciênc Saúde Coletiva 2003; 8:991-1003.

16. Araújo TM, Karasek R. Validity and reliability of the job content questionnaire in formal and informal jobs in Brazil. Scand J Work Environ Health Suppl 2008; (6):52-9.
17. Karasek RA. Job content questionnaire and user's guide. Lowell: University of Massachusetts; 1985.

18. Hosmer DW, Lemeshow S. Applied logistic regression. 2nd Ed. New York: John Wiley \& Sons; 2000.

19. Oliveira NF, Santana VS, Lopes AA. Razões de proporções e uso do método delta para intervalos de confiança em regressão logística. Rev Saúde Pública 1997; 31:90-9.

20. Hosmer DW, Hosmer T, Le Cessie S, Lemeshow S. A comparison of goodness-of-fit tests for the logistic regression model. Stat Med 1997; 16:965-80.

21. Saastamoinen P, Laaksonen M, Leino-Arjas P, Lahelma E. Psychosocial risk factors of pain among employees. Eur J Pain 2008; 13:102-8.

22. Araújo TM, Reis EJFB, Carvalho FM, Porto LA, Reis IC, Andrade JM. Fatores associados a alterações vocais em professoras. Cad Saúde Pública 2008; 24:1229-38.

23. Silvany-Neto A, Araújo TM, Dutra FR, Azi GR, Alves RL, Kavalkievicz C, et al. Condições de trabalho e saúde dos professores da rede particular de ensino de Salvador, Bahia. Rev Baiana Saúde Pública 2000; 24:42-56.

24. Delcor NS, Araújo TM, Reis EJFB, Porto LA, Carvalho FM, Silva MO, et al. Condições de trabalho e saúde dos professores da rede particular de ensino de Vitória da Conquista, Bahia, Brasil. Cad Saúde Pública 2004; 20:187-96.

25. Araújo TM, Sena IP, Viana MA, Araújo EM. Mal-estar docente: avaliação de condições de trabalho e saúde em uma instituição de ensino superior. Rev Baiana Saúde Pública 2005; 29:6-21.

26. Lorusso A, Bruno S, Caputo F, L'Abbate N. Musculoskeletal complaints among Italian X-ray technologists. Ind Health 2007; 45:705-8.

27. Smith DR, Wei N, Zhang Y-J, Wang RS. Musculoskeletal complaints and psychosocial risk factors among physicians in mainland China. Int J Ind Ergon 2006; 36:599-603.

28. Lipscomb H, Kucera K, Epling C, Dement J. Upper extremity musculoskeletal symptoms and disorders among a cohort of women employed in poultry processing. Am J Ind Med 2008; 51:24-36.

29. Hollmann S, Heuer H, Schmidt K-H. Control at work: a generalized resource factor for the prevention of musculoskeletal symptoms? Work Stress 2001; 15:29-39.

30. Fernandes RCP, Carvalho FM, Assunção AA, Silvany-Neto AM. Interactions between physical and psychosocial demands of work associated to low back pain. Rev Saúde Pública 2009; 43:326-34.

31. Bongers PM, Winter CR, Kompier MA, Hildebrandt VH. Psychosocial factors at work and musculoskeletal disease. Scand J Work Environ Health 1993; 19:297-312.

Recebido em 30/Jul/2010

Versão final reapresentada em 01/Mai/2011

Aprovado em 06/Mai/2011 